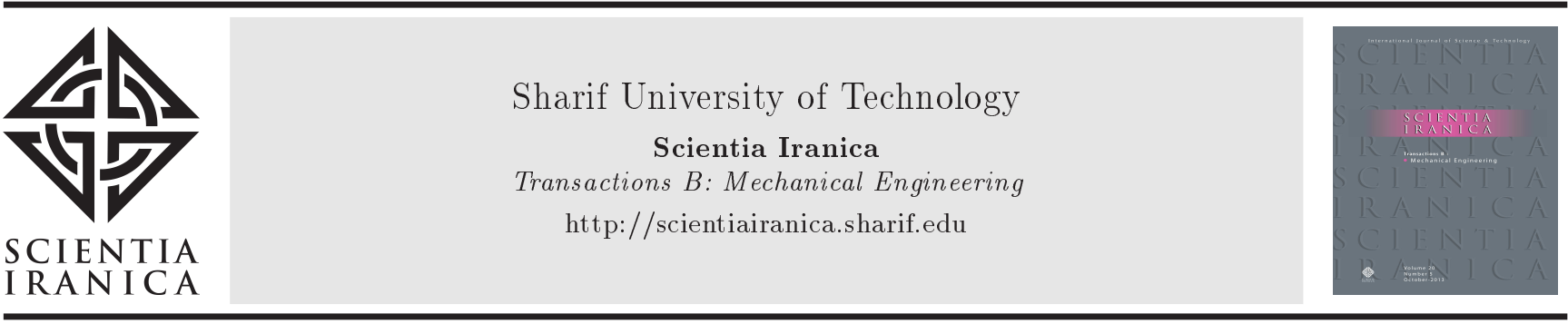

\title{
Vibration boundary control of micro-cantilever Timoshenko beam using piezoelectric actuators
}

\author{
A. Mehrvarz ${ }^{a}$, H. Salarieh ${ }^{a, *}$, A. Alasty ${ }^{a}$, and R. Vatankhah ${ }^{b}$ \\ a. School of Mechanical Engineering, Sharif University of Technology, Tehran, Iran. \\ b. School of Mechanical Engineering, Shiraz University, Shiraz, Iran. \\ Received 27 April 2016; received in revised form 3 January 2017; accepted 17 April 2017
}

\section{KEYWORDS}

Timoshenko micro beam;

Piezoelectric actuator;

PDE model;

Boundary control.

\begin{abstract}
One of the methods of force/moment exertion on micro beams is utilizing piezoelectric actuators. In this paper, considering the effects of the piezoelectric actuator on asymptotic stability achievement, the boundary control problem for the vibration of a clamped-free micro-cantilever Timoshenko beam is addressed. To achieve this purpose, the dynamic equations of the beam actuated by a piezoelectric layer laminated on one side of the beam are extracted. The control law was implemented so that vibrations of the beam could be decayed. This control law was achieved based on feedback of time derivatives of boundary states of the beam. The obtained control was applied in the form of piezoelectric voltage. To illustrate the impact of the proposed controller on the micro beam, the finiteelement method and Timoshenko beam element were used, and then simulation operation was performed. The simulation shows that not only does this control voltage reduce the vibration of the beam, but also the mathematical proofs proposed in this article are precise and implementable.
\end{abstract}

(C) 2018 Sharif University of Technology. All rights reserved.

\section{Introduction}

Nowadays, micro systems are one of the most useful tools in science and technology that are given special importance and status. The main function of these systems is based on the deformation of a beam at micro scale. Therefore, studying dynamic characteristics, behavior and control of the micro beams is of great significance. For example, using micro beams in Atomic Force Microscopy (AFM) [1], micro switches, mass sensors, micro-accelerometers, micro mirrors, determining a suitable position for construction at micro scale, Grating Light Valves (GLV) [2], can be noted.

For deforming micro beams, a suitable actuator is required. Among the most common actuators, elec-

\footnotetext{
*. Corresponding author.

E-mail address: salarieh@sharif.edu (H. Salarieh)
}

trostatic and piezoelectric actuators can be mentioned. Electrostatic actuators have so many applications such as Grating Light Valves (GLV) [2]. Another common category of actuators is piezoelectric actuators which are used for micro beams excitation. These actuators are made of piezoelectric materials. One of the most important properties of piezoelectric materials is their deformations caused by applying electric potential field. This property is used in piezoelectric actuators. In this method, a piezoelectric layer is attached to the micro beam. Since there is a constraint between this layer and the micro beam, increasing the length of the piezoelectric layer, due to applied voltage, will cause bending and deformation on the beam [3]. This method of stimulation is the basis of atomic force microscopy and is considered as the most effective tool in surface topography nowadays. The main application of this tool is the study of surface properties and manipulation of materials in nanoscale, surface modeling, assembling nanoparticles, and their communication [4-6]. 
Many studies on modeling micro beams and their associated applications have been performed; Grating Light Valves modeling is one example in which the micro beam works by an electrostatic excitation [7], and micro pump modeling is another instance where the micro beam is used as a displacement creator operator [8].

Many studies have been done in the field of micro systems analysis which include the study of the ideal micro beam model behavior $[9,10]$ and that of vibration analysis of beams $[11,12]$.

In addition to the issues raised in the modeling and analysis of micro beams, there have been comprehensive studies on control of beams. Based on the application where the micro beam is used, the target for the control of micro beam may change. For example, if the beam is used as a micro switch, the target would be the position control of the end of beam [13]. Moreover, atomic force microscopy can be considered as another example. Since the set of atomic force microscopy works in their resonant mode, the control objective is vibration control of the beam in their resonant mode [14]. Another important objective of control that is considerably significant in the control of micro beams is controlling the shape and position of the beam. For example, this issue has particular importance in the static atomic force microscopy [15].

Conventional control algorithms are usually suitable for controlling systems that have Ordinary Differential Equations (ODE). Such algorithms are not appropriate for continuum systems, such as beams and membranes. For this purpose, governing equations should be converted to ordinary differential equations in the vibrational modes, and then they would be controlled [3,16]. In contrast, in boundary control algorithm, the system is considered as a Partial Differential Equation (PDE) where the controller is applied onto the boundary of the system and can change the response by changing the boundary conditions of the system [17-19]. In this method, the boundary parameters are changed in such a way that the desirable behavior of the system is achieved eventually [2025]. This method may have a variety of applications in industry since the system is actuated only by its boundary and the controller is used only by the measured data from the boundary. For example, this method has been used to control the flexible marine riser [26-28] and flexible articulated wings on a Robotic Aircraft [29].

In this paper, boundary control of a clampedfree micro-cantilever Timoshenko beam with the aim of vibration suppression is considered. The actuator is a piezoelectric layer attached to the beam. Considering the effects of piezoelectric actuator, a boundary control is obtained which guarantees the asymptotic stability of the system. In this case, it is assumed that the piezoelectric layer is ideally attached to the beam. In the second section, the dynamic equations of the system are derived. In the third section, a linear control law based on the theory of boundary control is constructed to suppress the system vibration. In the fourth section, Finite-Element Method (FEM) is utilized for modeling the system. Simulation results before and after applying the control law are presented in the fifth section. Finally, conclusion is given in the last section.

\section{Model dynamics}

A clamped-free micro-cantilever Timoshenko beam is presented as the inspected beam to which a piezoelectric layer is ideally attached. $h^{b}$ is the beam thickness, $h^{p}$ is the piezoelectric thickness, $L$ is the length of beam, and $b$ is the width of beam, as shown in Figure 1.

Kinetic energy, $T$, and strain energy, $U$, of beams are achieved by Eqs. (1) and (2):

$$
\begin{aligned}
T= & \frac{1}{2} \int_{0}^{L}\left(A v_{t}^{2}+C \alpha_{t}^{2}\right) d x \\
U= & \frac{1}{2} \int_{0}^{L}\left(\varepsilon_{33} \frac{b^{p}}{h^{p}} u^{2}-k_{1} \alpha_{x} u-D \alpha_{x}^{2}\right. \\
& \left.-B\left(\nu_{x}-\alpha\right)^{2}\right) d x
\end{aligned}
$$

Partial differential equations of Timoshenko beam with a piezoelectric layer and boundary conditions obtained from the Hamilton principle with a little modification are given in [16]. A modification is made to the equations, that is, no external force has been entered into the system. The resulting equations are as follows:

$$
\begin{aligned}
& \left\{\begin{array}{l}
A \nu_{t t}-B\left(\nu_{x x}-\alpha_{x}\right)=0 \\
C \alpha_{t t}-D \alpha_{x x}-B\left(\nu_{x}-\alpha\right)=0
\end{array}\right. \\
& \left\{\begin{array}{l}
\alpha(0)=0 \\
\alpha_{x}(1)=-\frac{k_{1}}{D} u(t) \\
\nu(0)=0 \\
\nu_{x}(1)-\alpha(1)=0
\end{array}\right.
\end{aligned}
$$

where $x$ and $t$ indicate the independent spatial and time variables, respectively, $\nu(x, t)$ represents the lateral

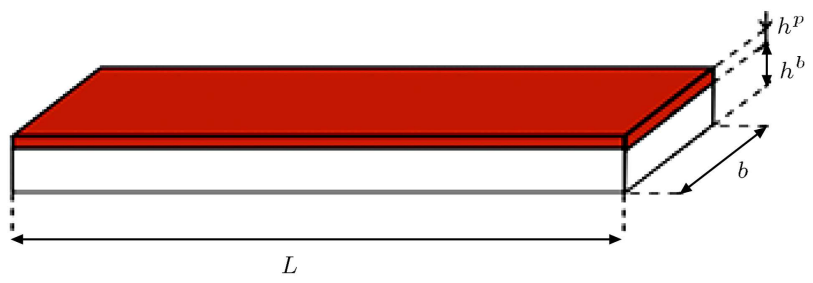

Figure 1. A schematic view of the beam with piezoelectric actuator and some geometric parameters [16]. 


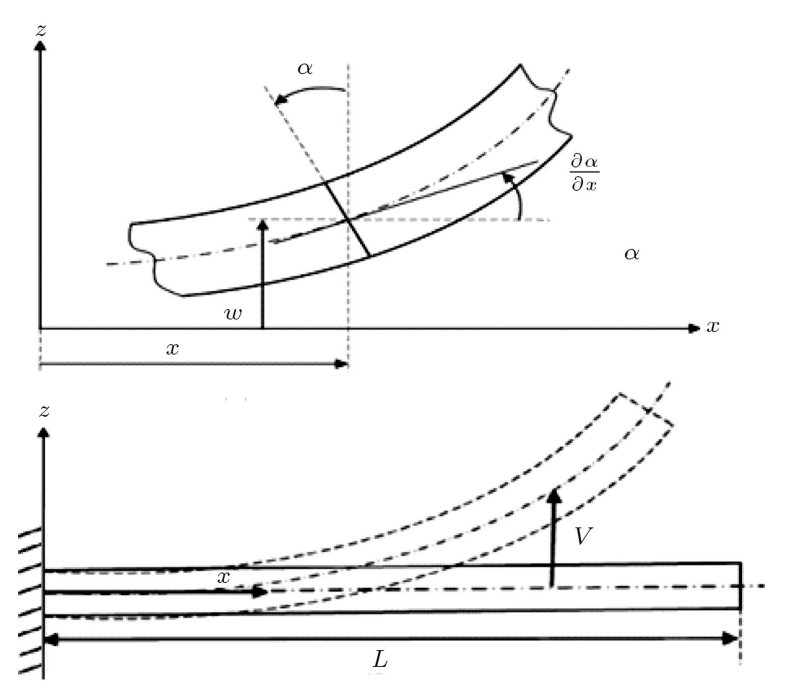

Figure 2. The coordinate system and variables of micro-cantilever Timoshenko beam [21].

deflection, and $\alpha(x, t)$ denotes the rotation of line elements along the centerline due to pure bending. The parameters and variables of Timoshenko beam are shown in Figure 2. Furthermore:

$$
\left\{\begin{array}{l}
A=\rho^{p} h^{p} b^{p}+\rho^{b} h^{b} b^{b} \\
B=4\left(k^{\prime} c_{55}^{p} h^{p} b^{p}+k^{\prime} b c_{55}^{b} h^{b} b^{b}\right) \\
C=\rho^{p} I^{p}+\rho^{b} I^{b} \\
D=c_{11}^{p} I^{p}+c_{11}^{b} I^{b} \\
k_{1}=e_{13} z_{m}^{p} b^{p}
\end{array}\right.
$$

In Eqs. (3) and (4), subscripts $x$ and $t$ indicate derivatives with respect to position and time. Moreover, in Eq. (5), superscripts $p$ and $b$ indicate that the desired parameter is related to the piezoelectric layer or beam; $z_{m}^{p}$ denotes the distance between the middle line of the piezoelectric layer and neutral axis of the beam; $I^{b}$ and $I^{p}$ denote the second moment of area of beam and piezoelectric layer; $\rho^{b}$ and $\rho^{p}$ denote the density of beam and piezoelectric layer, $c_{11}, c_{55}$, and $e_{13}$ are piezoelectric coefficients, and $k^{\prime}$ is the shape factor. For the rectangular cross-section, the value of shape factor will be obtained as follows [30]:

$$
k^{\prime}=\frac{10(1+v)}{12+11 v}
$$

where $v$ is the Poisson ratio.

$u(t)$ is the piezoelectric voltage in boundary condition (Eq. (4)) while, herein, it is the controlling force. The voltage appears only in the boundary condition, since it is assumed that the electrodes are attached perfectly and consistently onto the piezoelectric layer.

The main goal is designing a boundary controller for Timoshenko beam with the piezoelectric layer whose equations have been obtained in this section. In the next section, the boundary controller will be designed.

\section{Controller design}

The purpose of designing a vibration suppression controller is to stabilize vibrations of the system caused by initial velocity or displacement of the micro beam. Some conventional methods are available to design a controller for a system whose governing equation is partial differential equation; one of these methods is to convert these equations into several ordinary differential ones. In this case, practically, the main model changes, some information derived from the system may be lost, and the system is investigated only in a few specific modes. In contrast, in the method used in this paper, the controller is designed without changing the equations of the system by considering the Lyapunov theory and using boundary control method.

\subsection{Boundary control}

Knowing well-posedness of the closed-loop system has great importance in this investigation, especially when a boundary control is used for eliminating the system vibration. Therefore, in this case, well-posedness of the controlled clamped-free micro-cantilever Timoshenko model should be investigated. To this end, semigroup technique and operator theory should be used. After that, the asymptotic stability of the closed-loop system will be proved by utilizing the Lyapunov stability method and LaSalle's invariant set theorem.

For boundary controller design and wellposedness analysis of the controlled system, the PDEs model of Eq. (3) should be obtained in the state-space representation as follows:

$$
X_{t}=[A]_{4 \times 4} X .
$$

In Eq. (7):

$$
\begin{aligned}
& X=\left[\begin{array}{c}
\nu \\
\nu_{t} \\
\alpha \\
\alpha_{t}
\end{array}\right], \\
& {[A]=\left[\begin{array}{cccc}
0 & 1 & 0 & 0 \\
a_{\nu} & 0 & a_{\alpha} & 0 \\
0 & 0 & 0 & 1 \\
b_{\nu} & 0 & a_{\alpha} & 0
\end{array}\right],}
\end{aligned}
$$

and in Eq. (9):

$$
\left\{\begin{array}{l}
a_{\nu}=\frac{B}{A} \frac{\partial^{2}}{\partial x^{2}} \\
a_{\alpha}=-\frac{B}{A} \frac{\partial}{\partial x} \\
b_{\nu}=\frac{B}{C} \frac{\partial}{\partial x} \\
b_{\alpha}=\frac{D}{C} \frac{\partial^{2}}{\partial x^{2}}-\frac{B}{C}
\end{array}\right.
$$

In Eq. (7), matrix $A$ is defined as the PDE's operator of the problem. To achieve our control target, a proper functional space and its corresponding inner 
product should be defined due to the kinetic and strain energies of the system represented in Eqs. (1) and (2). The appropriate functional space for our problem in occupying region $\Omega$ is chosen as follows:

$$
V=H^{2}(\Omega) \times L^{2}(\Omega) \times H^{2}(\Omega) \times L^{2}(\Omega),
$$

where $L^{p}(\Omega)$ is a Lebesgue space (space of functions with the property of $\left.\left[\int_{\Omega}|f|^{p} d \mu\right]^{\frac{1}{p}}<\infty\right) ; H^{k}(\Omega)$ is a Hilbert space that addresses Sobolev space, $W^{k 2}(\Omega)$, of functions $W^{k 2}(\Omega) \equiv H^{k}(\Omega)\left\{f: D^{\alpha} f \in\right.$ $L^{2}(\Omega)$, for all $\left.0 \leq \alpha \leq k\right\}$, where $D^{\alpha} . f$ is the $\alpha$ th-order weak derivative of function $f$ [31]. The corresponding inner product introduced in Hilbert space $V$ has the following form:

$$
\begin{aligned}
\langle Y, Z\rangle= & \frac{1}{2} \int_{\Omega}\left(A a_{2} b_{2}+C a_{4} b_{4}+D a_{3 x} b_{3 x}\right. \\
& \left.+B\left(a_{1 x}-a_{3}\right)\left(b_{1 x}-b_{3}\right)\right) d \Omega
\end{aligned}
$$

where $Y=\left(a_{1}, a_{2}, a_{3}, a_{4}\right), Z=\left(b_{1}, b_{2}, b_{3}, b_{4}\right)$, and $a_{i}, b_{i}$ for $i=1,2, \ldots, 4$ are scalar-valued functions defined on $\Omega$, where $a_{j}, b_{j} \in H^{2}(\Omega), j=1,3 ; a_{j}, b_{j} \in L^{2}(\Omega)$, $j=2,4$. Eq. (11) can be used to define the mechanical energy of closed-loop system. The target of this investigation is to show that System (3), with boundary conditions of Eq. (4), which appeared in the following equation under boundary feedbacks, is well-posed and has an asymptotic decay rate:

$$
u(t)=k_{u} \alpha_{t}(L) .
$$

In Eq. (12), $k_{u}$ is the controller gain and has a positive value. This controller is applied to piezoelectric layer as voltage to reduce vibrations of Timoshenko micro beam.

The state space representation of the system in Eqs. (3) and (4), under boundary controllers shown in Eq. (12), is summarized as follows:

$$
\left\{\begin{array}{l}
X_{t}=[A] X \\
\Gamma_{0}: x=0 \rightarrow \nu=\nu_{x}=\alpha=\alpha_{x}=0 \\
\Gamma_{L}: x=L \rightarrow \alpha_{x}(L)=-\frac{k_{1}}{D} u(t), \nu_{x}(L)=\alpha(L)
\end{array}\right.
$$

Considering operator $A$ and boundary conditions of the system in Eq. (13), the domain of operator $A$ is determined as: $D(A)=H_{\Gamma_{0}}^{4}(\Omega) \times H^{2}(\Omega) \times H_{\Gamma_{0}}^{4}(\Omega) \times$ $H^{2}(\Omega)$ and:

$$
H_{\Gamma_{0}}^{4}(\Omega)=\left\{f: f \in H^{4}(\Omega),\left.f\right|_{\Gamma_{0}}=\left.f_{x}\right|_{\Gamma_{0}}\right\} .
$$

To illustrate well-posedness of the controlled system expressed in Eq. (13), first, it should be demonstrated that operator $A$ is a dissipative operator.

Theorem 3.1. Linear operator $A$, whose domain is defined in Eq. (14), is dissipative.
Proof. From the definition of the inner product in Eq. (11), we have:

$$
\begin{aligned}
\langle X, X\rangle_{V}= & \frac{1}{2} \int_{0}^{L}\left[A \nu_{t}^{2}+C \alpha_{t}{ }^{2}+D \alpha_{x}{ }^{2}\right. \\
& \left.+B\left(v_{x}-\alpha\right)^{2}\right] d x=E(t) .
\end{aligned}
$$

The following result is achieved after applying the time derivative to the above-mentioned positive definite function which can be considered as a Lyapunov function:

$$
\begin{aligned}
& \frac{d}{d t}\langle X, X\rangle_{\nu}= 2\langle X, A X\rangle_{\nu}=\int_{0}^{L}\left[A \nu_{t t} \nu_{t}+C \alpha_{t} \alpha_{t t}\right. \\
&\left.+D \alpha_{x} \alpha_{x t}+B\left(\nu_{x}-\alpha\right)\left(\nu_{x t}-\alpha_{t}\right)\right] d x \\
& d(16)
\end{aligned}
$$

By replacing $\nu_{t t}$ and $\alpha_{t t}$ from Eq. (3), we have:

$$
\begin{aligned}
\langle X, A X\rangle_{\nu}= & \frac{1}{2} \int_{0}^{L}\left[B \nu_{t}\left(\nu_{x x}-\alpha_{x}\right)\right. \\
& +\alpha_{t}\left(D \alpha_{x x}+B \nu_{x}-B \alpha\right)++D \alpha_{x} \alpha_{x t} \\
& \left.+B \nu_{x} \nu_{x t}-B \nu_{x} \alpha_{t}-B \alpha \nu_{x t}+B \alpha \alpha_{t}\right] d x
\end{aligned}
$$

With arranging Eq. (17), we have:

$$
\begin{aligned}
\langle X, A X\rangle_{\nu}= & \frac{1}{2} \int_{0}^{L}\left[B\left[\left(\nu_{t} \nu_{x x}+v_{x} \nu_{x t}\right)+\left(-\alpha_{x} \nu_{t}-\alpha \nu_{x t}\right)\right]\right. \\
& \left.+D\left(\alpha_{t} \alpha_{x x}+\alpha_{x} \alpha_{x t}\right)\right] d x
\end{aligned}
$$

Performing some integration by parts, the following results are achieved:

$$
\begin{aligned}
\langle X, A X\rangle_{\nu}= & \left.\frac{1}{2}\left[B \nu_{t} \nu_{x}-B \nu_{t} \alpha+D \alpha_{x} \alpha_{t}\right]\right|_{0} ^{L}, \\
\langle X, A X\rangle_{\nu}= & \frac{1}{2}\left[B \nu t(L) \nu_{x}(L)-B \nu_{t}(0) \nu_{x}(0)\right. \\
& -B \nu_{t}(L) \alpha(L)+B \nu_{t}(0) \alpha(0) \\
& \left.+D \alpha_{x}(L) \alpha_{t}(L)-D \alpha_{x}(0) \alpha_{t}(0)\right] .
\end{aligned}
$$

By applying the boundary conditions of Eq. (4), we have:

$$
\langle X, A X\rangle_{\nu}=-\frac{1}{2} k_{1} \alpha_{t}(L) u(t) .
$$

Finally, by applying boundary control laws of Eq. (12), the following result can be obtained: 


$$
\langle X, A X\rangle_{\nu}=-\frac{1}{2} k_{1} k_{u} \alpha_{t}^{2}(L)
$$

According to Eq. (22), it is concluded that $\langle X, A X\rangle_{V} \leq$ 0 for the closed-loop system of Eq. (13). Thus, from the definition of the dissipative operators [32], the proof will be completed.

Theorem 3.2. The operator $(\gamma I-A)^{-1}$ exists and is continuous for any $\gamma>0$.

Proof. We consider the following equation:

$$
(\gamma \mathrm{I}-A) X=X_{0}
$$

For demonstrating the existence of the operator $(\gamma I-$ $A)^{-1}$, it is sufficient to show that there is only one solution to Eq. (23). By applying the result of Theorem 3.1 illustrated in Eq. (22), we have:

$$
\begin{aligned}
\langle(\gamma I-A) X, X\rangle_{V} & =\langle\gamma X, A X\rangle_{V}-\langle A X, X\rangle_{V} \\
& =\gamma\langle X, X\rangle_{V}+\frac{1}{2} k_{1} k_{u} \alpha_{t}^{2}(L) \\
& \geq \gamma\langle X, X\rangle_{V}=\gamma\|X\|_{V}^{2}
\end{aligned}
$$

With the above result, it is concluded that the bilinear form $a$ with the definition of $a(u, \nu)=\langle(\gamma I-A) u, v\rangle$ is coercive on the Hilbert space, $V$. Now, using the LaxMilgram theorem, one can easily prove that Eq. (23) has a unique weak solution, and so the operator $(\gamma I-$ $A)^{-1}$ exists [33,34].

It is shown that the operator $(\gamma I-A)^{-1}$ is bounded and, as given in [31], it will be continuous. Thus, in order to complete the proof, it is sufficient to show that the operator $(\gamma I-A)^{-1}$ is bounded. As shown, Inequality (24) is attained from the dissipativity of operator $A$. Now, one can conclude that:

$$
\begin{aligned}
\gamma\|X\|_{V}^{2} & \leq\langle(\gamma I-A) X, X\rangle_{V}=\left\langle X_{0}, X\right\rangle \\
& \leq\left\|X_{0}\right\|_{V}\|X\|_{V} \rightarrow\left\|X_{0}\right\|_{V} \geq \gamma\|X\|_{V}
\end{aligned}
$$

So, $\|X\|_{V}$ is bounded when $\left\|X_{0}\right\|_{V}$ is bounded and the proof is completed.

Theorem 3.3. The set of Eq. (13), with the initial condition $X(t=0) \in D(A)$, is well-posed.

Proof. Since the closure of function space, $D(A)$, is $H^{4}(\Omega) \times H^{2}(\Omega) \times H^{4}(\Omega) \times H^{2}(\Omega) \subset V$, it is clear that $D(A)$ is dense in $V$. It is evidential that range of $(\gamma I-A), \mathcal{R}(\gamma I-A)=V$ is dense in $V$. Also, according to Theorem 3.2, $(\gamma I-A)$ has a continuous inverse $(\gamma I-A)^{-1}$ for any $\gamma>0$. Therefore, according to the definition of resolving the set of an operator [32], $\gamma$ is in the resolving set of operator $A$.
As shown in Theorem 3.1, it is demonstrated that operator $A$ is a dissipative operator. Therefore, based on the Lumer-Phillips theorem [34], the system presented in Eq. (13), containing the boundary controllers of Eq. (12), with any initial condition $X(t=0) \in$ $D(A)$, is well-posed.

The asymptotic stability of the closed-loop system is achieved using LaSalle's invariant set theorem, which is based on the Lyapunov method. For this target, first, it is required to show that the operator $(\gamma I-A)^{-1}$ is compact for any $\gamma>0$ [35].

Theorem 3.4. The operator $(\gamma I-A)^{-1}$ is compact for any $\gamma>0$.

Proof. It should be proved that the operator $(\gamma I-$ $A)^{-1}$ is bounded for any $\gamma>0$ [32]. This subject is shown in the proof of Theorem 3.2. Also, it is obvious that:

$$
(\gamma I-A)^{-1} V \in D(A)=D(A) .
$$

Since the closure of $(\gamma I-A)^{-1} V$ is $H^{4}(\Omega) \times H^{2}(\Omega) \times$ $H^{4}(\Omega) \times H^{2}(\Omega)$ and this space is compactly embedded in $H^{2}(\Omega) \times L^{2}(\Omega) \times H^{2}(\Omega) \times L^{2}(\Omega)$ [32], according to Rellich-Kondrachov compact embedding theorem [32], the compactness of the above-mentioned resolving set is obtained and the proof will be completed.

Now, the proof of the asymptotic stability of the closed-loop system using LaSalle's invariant set theorem would be performed.

Theorem 3.5. The states of the closed-loop system of Eq. (13) with the boundary feedback control laws of Eq. (12) will tend asymptotically toward zero.

Proof. We introduce the following Lyapunov functional candidate, which is the mechanical energy of the closed-loop system, $E(t)=\langle X, X\rangle_{V} \geq 0$. As shown in the proof of Theorem 3.1, time derivative of the above functional is derived as:

$$
\dot{E}(t)=-k_{1} k_{u} \alpha_{t}^{2}(L),
$$

where the superimposed dot indicates differentiation with respect to time $t$. One can easily see from Eq. (27) that $\dot{E}(t) \leq 0$. Accordingly, function $E(t)$ admits the requirements of a Lyapunov function. At this step, because of the compactness of resolving $(\gamma I-A)^{-1}$ proved in Theorem 3.4, the LaSalle invariant set theorem [35] gives the asymptotic decay rate of the controlled states, and the proof will be completed.

In this section, stability of the Timoshenko beam with piezoelectric layer has been proved. Mathematical proof is sufficient for showing the asymptotic stability of the proposed controlled system. However, numerical simulation has been presented to show the accuracy and applicability of the proposed method. 


\section{Finite-element model}

This section discusses the issue of modeling the beam by considering the Timoshenko beam element. It is assumed that the element has two nodes, and each node has the following variables:

$\left[\begin{array}{ll}\nu & \alpha\end{array}\right]$

$\nu$ and $\alpha$ have the following polynomial forms:

$$
\left\{\begin{array}{l}
v=c_{1}+c_{2} x \\
\alpha=c_{3}+c_{4} x
\end{array}\right.
$$

Eq. (28) can be written in a matrix form as in Eq. (29):

$$
\begin{aligned}
{\left[\begin{array}{l}
\nu \\
\alpha
\end{array}\right] } & =\left[\begin{array}{llll}
1 & x & 0 & 0 \\
0 & 0 & 1 & x
\end{array}\right]\left[\begin{array}{l}
c_{1} \\
c_{2} \\
c_{3} \\
c_{4}
\end{array}\right]=g C=g h^{-1} q \\
& =g\left[\begin{array}{cccc}
1 & 0 & 0 & 0 \\
0 & 0 & 1 & 0 \\
1 & L_{e} & 0 & 0 \\
0 & 0 & 1 & L_{e}
\end{array}\right]^{-1}\left[\begin{array}{l}
\nu_{1} \\
\alpha_{1} \\
\nu_{2} \\
\alpha_{2}
\end{array}\right]=N q
\end{aligned}
$$

For obtaining constant parameters $c_{i}, i=1, \ldots, 4, h$ matrix in two points $x=0, L_{e}$ is defined. In addition, $L_{e}$ indicates the length of the beam element.

In Eq. (29), $N$ is the shape function and will be defined as in Eq. (30):

$$
N=g h^{-1}=\left[\begin{array}{cccc}
H_{1} & 0 & H_{2} & 0 \\
0 & H_{1} & 0 & H_{2}
\end{array}\right]
$$

$H_{i}, i=1,2$, is defined as in Eq. (31):

$$
\left\{\begin{array}{l}
H_{1}=1-\frac{x}{L_{e}} \\
H_{2}=\frac{x}{L_{e}}
\end{array}\right.
$$

By using kinetic and strain energy of Systems (1) and (2), and variational method [36], the mass, stiffness, and force matrices will be obtained as in Eqs. (32)-(34), respectively:

$$
\begin{aligned}
& K_{e}=\int_{0}^{L_{e}}\left(B_{1}^{T} D B_{1}+B_{2}^{T} B B_{2}\right) d x \\
& M_{e}=\int_{0}^{L_{e}}\left(D_{1}^{T} A D_{1}+D_{2}^{T} C D_{2}\right) d x \\
& F_{e}=\int_{0}^{L_{e}}-\frac{1}{2} k_{1} U B_{1}^{T} d x .
\end{aligned}
$$

As a result, $D_{i}$ and $B_{i}, i=1,2$, are:

$$
\left\{\begin{array}{l}
B_{1}=\left[\begin{array}{ll}
0 & \frac{\partial}{\partial x}
\end{array}\right] N \\
B_{2}=\left[\begin{array}{ll}
\frac{\partial}{\partial x} & -1
\end{array}\right] N \\
D_{1}=\left[\begin{array}{ll}
1 & 0
\end{array}\right] N \\
D_{2}=\left[\begin{array}{ll}
0 & 1
\end{array}\right] N
\end{array}\right.
$$

By replacing and integrating matrices, Eqs. (36)-(38) will be obtained as follows:

$$
\begin{aligned}
& K_{e}=\left[\begin{array}{cccc}
\frac{B}{L_{e}} & \frac{B}{2} & -\frac{B}{L_{e}} & \frac{B}{2} \\
\frac{B}{2} & \frac{D}{L_{e}}+\frac{B L_{e}}{3} & -\frac{B}{2} & \frac{B L_{e}}{6}-\frac{D}{L_{e}} \\
-\frac{B}{L_{e}} & -\frac{B}{2} & \frac{B}{L_{e}} & -\frac{B}{2} \\
\frac{B}{2} & \frac{B L_{e}}{6}-\frac{D}{L_{e}} & -\frac{B}{2} & \frac{D}{L_{e}}+\frac{B L_{e}}{3}
\end{array}\right], \\
& M_{e}=\left[\begin{array}{cccc}
\frac{A L_{e}}{3} & 0 & \underline{A L_{e}} & 0 \\
\frac{C L_{e}}{A L_{e}} & 6 & \underline{A L_{e}} & 0 \\
6 & \frac{C L_{e}}{3} & 3 & \underline{C L_{e}} \\
0 & 6 & 0 & 3
\end{array}\right], \\
& F_{e}=\left[\begin{array}{c}
0 \\
\frac{k_{1} U}{2} \\
0 \\
-\frac{k_{1} U}{2}
\end{array}\right] \text {. }
\end{aligned}
$$

Here, we assume that ten nodes for our system and matrices $M, K$, and $F$ will be obtained by assembling the matrices given by Eqs. (36)-(38). Time evolution of the system will be obtained by numerical integration of Eq. (39) as follows:

$$
[M]\{\ddot{q}\}+[K]\{q\}=\{F\} .
$$

\section{Simulation}

In this section, the simulation operation with regard to System (1) has been performed with boundary condition in Eq. (2). A proper control gain obtained from trial and error which has a suitable settling 
Table 1. Material properties of silicon dioxide for beam and PZT for actuator.

\begin{tabular}{ccc}
\hline & \multicolumn{2}{c}{ Material } \\
\cline { 2 - 3 } & $\mathrm{SiO}_{\mathbf{2}}$ & $\mathbf{P Z T}$ \\
\hline Density $(\boldsymbol{\rho})\left(\mathrm{kg} / \mathbf{m}^{3}\right)$ & 2200 & 7700 \\
Poisson coefficient $(\boldsymbol{\nu})$ & 0.17 & 0.31 \\
Young modulus of elasticity $(\boldsymbol{E})(\mathbf{G P a})$ & 73 & 71 \\
Piezoelectric constants $(\mathbf{1 0 - 1 2} \mathbf{C} / \mathbf{N})$ & - & $d_{31}=175$ \\
Relative permittivity & & $d_{33}=400$ \\
& & $d_{55}=580$ \\
\end{tabular}

time and transient response is considered as $k_{u}=0.2$. It should be mentioned that by increasing the value of controller gain, vibrations of the beam decrease rapidly; however, it causes numerical problems due to stiffness of equations and severe slopes in velocity and displacement variables, which affect the simulation results. Indeed, $k_{u}=0.2$ is the most suitable gain, which can be utilized in our simulations without any numerical difficulty. A silicon dioxide micro cantilever with a PZT layer laminated on one of its side is considered as a case study. The physical characteristics of the beam and piezoelectric layer can be found in Table $1[2,16,37]$. According to Table 1:

$$
e_{13}=\sum_{1}^{3} d_{3 i} c_{1 i}=-3.621 \mathrm{C} / \mathrm{m}^{2}
$$

The geometry of the piezoelectric layer and beam is given in Table 2 [16].

Using the following definitions of parameters, the

Table 2. Geometrical dimensions of beam and piezoelectric layer (all in $\mu \mathrm{m}$ ).

\begin{tabular}{cc}
\hline Beam length $(L)$ & 90 \\
Beam thickness $\left(h^{b}\right)$ & 10 \\
Beam width $\left(b^{b}\right)$ & 30 \\
Piezoelectric thickness $\left(h^{p}\right)$ & 10 \\
Piezoelectric width $\left(b^{p}\right)$ & 30 \\
\hline
\end{tabular}

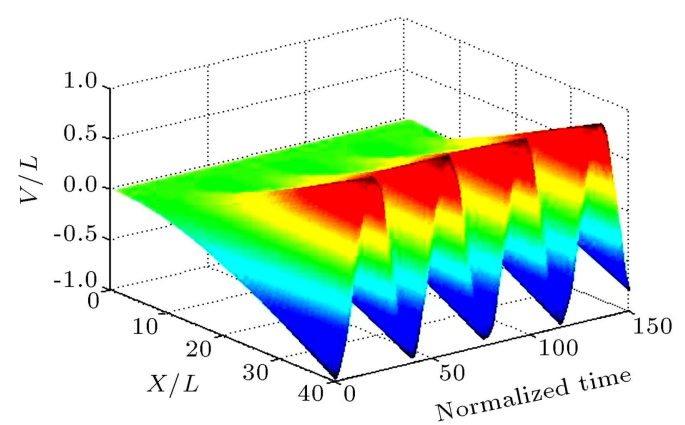

(a) governing equation of the system will become nondimensionalized:

$$
\left\{\begin{array}{lll}
\tilde{x}=\frac{x}{L} & \tilde{\rho}^{b}=\frac{\rho^{b}}{\rho^{b}} \quad \tilde{e}_{13}=\frac{e_{13}}{e_{13}} \\
\tilde{L}=\frac{L}{L} \quad \tilde{\rho}^{p}=\frac{\rho^{p}}{\rho^{b}} \quad \tilde{c}_{1}=\frac{c_{1}}{\rho^{b} L^{2} \omega_{1}} \\
\tilde{b}=\frac{b}{L} \quad \tilde{I}^{b}=\frac{I^{b}}{L^{4}} \quad \tilde{c}_{2}=\frac{c_{2}}{\rho^{b} L^{2} \omega_{1}} \\
\tilde{h}^{p}=\frac{h^{p}}{L} \quad \tilde{I}^{p}=\frac{I^{p}}{L^{4}} \quad \tilde{u}=\frac{u e_{13}}{\rho^{b} L^{3} \omega_{1}^{2}} \\
\tilde{h}^{b}=\frac{h^{b}}{L} \quad \tilde{c}_{i j}=\frac{c_{i j}}{\rho^{b} L^{2} \omega_{1}^{2}} \quad \tilde{t}=t \omega_{1}
\end{array}\right.
$$

Considering that there are ten nodes in the beam and utilizing the information available in Tables 1 and 2, 40 ordinary differential equations should be numerically solved at the same time. At first, it is assumed that no force enters to the system, and in Eq. (39), $F$ matrix is set to zero. The micro beam behavior is shown in Figure 3. Then, it is assumed that the system is closed loop, and there is controlling force on the beam obtained from the piezo-electric actuator. The behavior is shown in Figure 4. The control signal is used as the piezoelectric voltage shown in Figure 5.

As it is clear, after applying the control action, vibrations of the system are suppressed and the system becomes asymptotically stable.

\section{Conclusion}

In this paper, the governing partial differential equations and corresponding boundary conditions of micro-

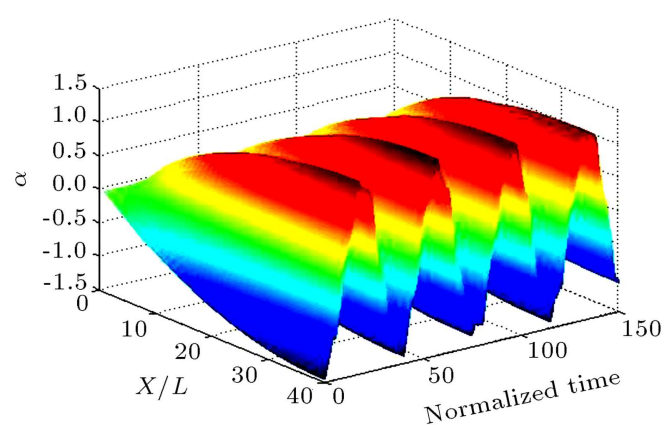

(b)

Figure 3. Open-loop response of the micro beam: (a) Lateral deflection $\nu(x, t)$ and (b) rotation of line elements along the centerline $\alpha(x, t)$. 


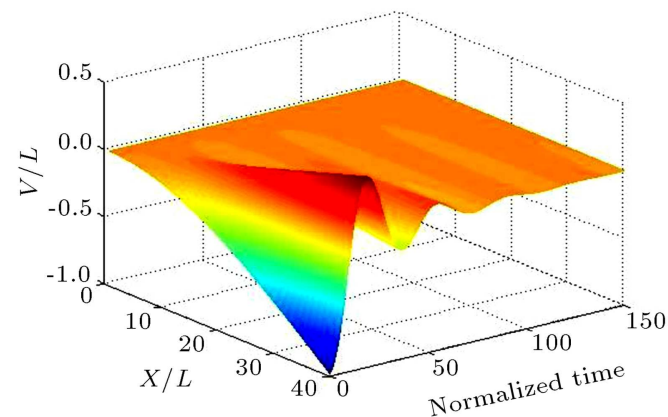

(a)

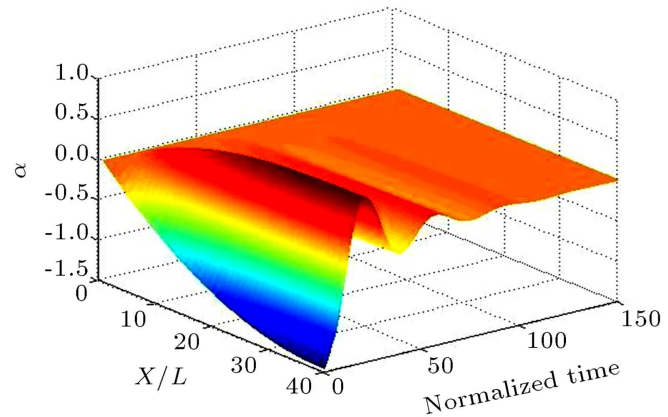

(b)

Figure 4. Closed-loop response of the micro beam: (a) Lateral deflection $\nu(x, t)$ and (b) rotation of line elements along the centerline $\alpha(x, t)$.

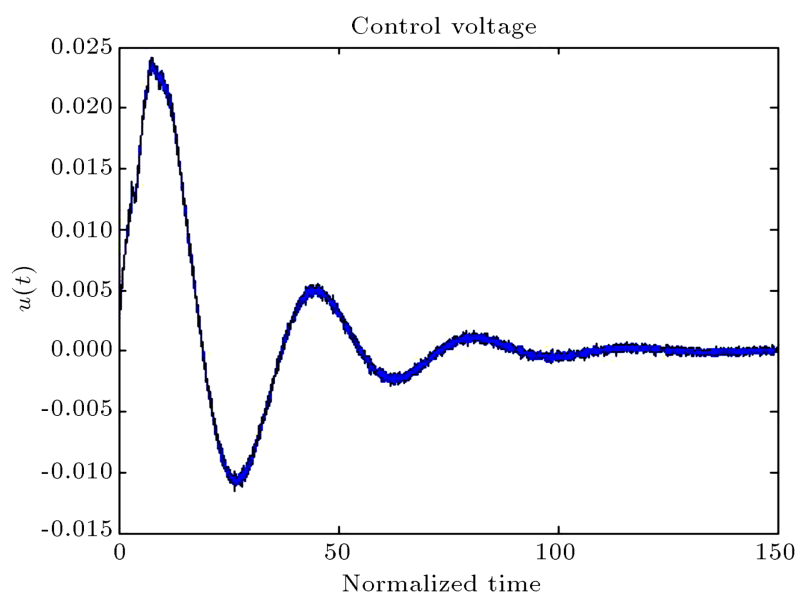

Figure 5. Control voltage $u(t)=k_{u} \alpha_{t}(L)$.

cantilever Timoshenko beams were obtained. Transverse vibration of the micro beam was stabilized by designing proper boundary control laws. This control law was achieved from the feedback of time derivatives of boundary states of the beam. The obtained control was applied in the form of voltage of the piezoelectric actuator. Boundary stabilization was investigated using the Lyapunov stability method and LaSalle's invariant set theorem. To demonstrate the performance of the designed controllers via numerical simulation, the finite-element method was used to simulate the open-loop and closed-loop systems. In the FEM, the Timoshenko beam element was used. At the end, computer simulation results verified the achieved theoretical results of this work.

\section{References}

1. Indermühle, P., Schürmann, G., Racine, G., and De Rooij, N. "Atomic force microscopy using cantilevers with integrated tips and piezoelectric layers for actuation and detection", Journal of Micromechanics and Microengineering, 7(3), p. 218 (1997).

2. Maluf, N. and Williams, K., Introduction to Microelectromechanical Systems Engineering, Artech House (2004).
3. Zhang, W., Meng, G., and Li, H. "Adaptive vibration control of micro-cantilever beam with piezoelectric actuator in MEMS", The International Journal of Advanced Manufacturing Technology, 28(3-4), pp. 321327 (2006).

4. Gåhlin, R. and Jacobson, S. "A novel method to map and quantify wear on a micro-scale", Wear, 222(2), pp. 93-102 (1998).

5. García, R., Calleja, M., and Pérez-Murano, F. "Local oxidation of silicon surfaces by dynamic force microscopy: Nanofabrication and water bridge formation", Applied Physics Letters, 72(18), pp. 2295-2297 (1998).

6. Miyahara, K., Nagashima, N., Ohmura, T., and Matsuoka, S. "Evaluation of mechanical properties in nanometer scale using AFM-based nanoindentation tester", Nanostructured Materials, 12(5), pp. 10491052 (1999).

7. Furlani, E. "Simulation of grating light valves", in Technical Proceeding of the 1998 International Conference on Modeling and Simulation of Microsystems (1998).

8. Arik, M., Zurn, S., Bar-Cohen, A., Nam, Y., Markus, D., and Polla, D. "Development of CAD model for MEMS micropumps", in Technical Proceedings of the 1999 International Conference on Modeling and Simulation of Microsystems (1999).

9. Bernstein, D., Guidotti, P., and Pelesko, J. "Mathematical analysis of an electrostatically actuated MEMS device", Proceedings of the Modeling and Simulation of Microsystems MSM, pp. 489-492 (2000).

10. Liu, J., Mei, Y., Xia, R., and Zhu, W. "Large displacement of a static bending nanowire with surface effects", Physica E: Low-Dimensional Systems and Nanostructures, 44(10), pp. 2050-2055 (2012).

11. Alhazza, K.A., Daqaq, M.F., Nayfeh, A.H., and Inman, D.J. "Non-linear vibrations of parametrically excited cantilever beams subjected to non-linear 
delayed-feedback control", International Journal of Non-Linear Mechanics, 43(8), pp. 801-812 (2008).

12. Zhao, D., Liu, J., and Wang, L. "Nonlinear free vibration of a cantilever nanobeam with surface effects: Semi-analytical solutions", International Journal of Mechanical Sciences, 113, pp. 184-195 (2016).

13. McCarthy, B., Adams, G.G., McGruer, N.E., and Potter, D. "A dynamic model, including contact bounce, of an electrostatically actuated microswitch", Journal of, Microelectromechanical Systems, 11(3), pp. 276-283 (2002).

14. Jalili, N. and Laxminarayana, K. "A review of atomic force microscopy imaging systems: application to molecular metrology and biological sciences", Mechatronics, 14(8), pp. 907-945 (2004).

15. Krstic, M., Guo, B.-Z., Balogh, A., and Smyshlyaev, A. "Control of a tip-force destabilized shear beam by observer-based boundary feedback", SIAM Journal on Control and Optimization, 47(2), pp. 553-574 (2008).

16. Shirazi, M.J., Salarieh, H., Alasty, A., and Shabani, R. "Tip tracking control of a micro-cantilever Timoshenko beam via piezoelectric actuator", Journal of Vibration and Control, 19(10), pp. 1561-1574 (2013).

17. Canbolat, H., Dawson, D., Rahn, C., and Vedagarbha, P. "Boundary control of a cantilevered flexible beam with point-mass dynamics at the free end", Mechatronics, 8(2), pp. 163-186 (1998).

18. Dogan, M. and Morgul, O. "Boundary control of a rotating shear beam with observer feedback", Journal of Vibration and Control, 18(14), pp. 2257-2265 (2011).

19. Fard, M. and Sagatun, S. "Exponential stabilization of a transversely vibrating beam via boundary control", Journal of Sound and Vibration, 240(4), pp. 613-622 (2001).

20. Sadek, I., Kucuk, I., Zeini, E., and Adali, S. "Optimal boundary control of dynamics responses of piezo actuating micro-beams", Applied Mathematical Modelling, 33(8), pp. 3343-3353 (2009).

21. Vatankhah, R., Najafi, A., Salarieh, H., and Alasty, A. "Asymptotic decay rate of non-classical strain gradient Timoshenko micro-cantilevers by boundary feedback", Journal of Mechanical Science and Technology, 28(2), pp. 627-635 (2014).

22. He, W., Ge, S.S., How, B.V.E., Choo, Y.S., and Hong, K.-S. "Robust adaptive boundary control of a flexible marine riser with vessel dynamics", Automatica, 47(4), pp. 722-732 (2011).

23. Yang, K.-J., Hong, K.-S., and Matsuno, F. "Boundary control of a translating tensioned beam with varying speed", Mechatronics, IEEE/ASME Transactions on, 10(5), pp. 594-597 (2005).
24. Vatankhah, R., Najafi, A., Salarieh, H., and Alasty, A. "Boundary stabilization of non-classical micro-scale beams", Applied Mathematical Modelling, 37(20), pp. 8709-8724 (2013).

25. Vatankhah, R., Najafi, A., Salarieh, H., and Alasty, A. "Exact boundary controllability of vibrating nonclassical Euler-Bernoulli micro-scale beams", Journal of Mathematical Analysis and Applications, 418(2), pp. 985-997 (2014).

26. He, W., Ge, S.S., and Zhang, S. "Adaptive boundary control of a flexible marine installation system", Automatica, 47(12), pp. 2728-2734 (2011).

27. How, B., Ge, S., and Choo, Y. "Active control of flexible marine risers", Journal of Sound and Vibration, 320(4), pp. 758-776 (2009).

28. Nguyen, T., Do, K.D., and Pan, J. "Boundary control of coupled nonlinear three dimensional marine risers", Journal of Marine Science and Application, 12(1), pp. 72-88 (2013).

29. Paranjape, A.A., Guan, J., Chung, S.-J., and Krstic, M. "PDE boundary control for flexible articulated wings on a robotic aircraft", Robotics, IEEE Transactions on, 29(3), pp. 625-640 (2013).

30. Han, S.M., Benaroya, H., and Wei, T. "Dynamics of transversely vibrating beams using four engineering theories", Journal of Sound and Vibration, 225(5), pp. 935-988 (1999).

31. Reddy, J.N., Applied Functional Analysis and Variational Methods in Engineering, McGraw-Hill College (1986).

32. Robinson, J.C., Infinite-Dimensional Dynamical Systems: An Introduction to Dissipative Parabolic PDEs and the Theory of Global Attractors, 28, Cambridge University Press (2001).

33. Yosida, K., Functional Analysis, Springer (1980).

34. Pazy, A., Semigroups of Linear Operators and Applications to Partial Differential Equations, 44, Springer New York (1983).

35. Guo, B.-Z. and Morgül, Ö., Stability and Stabilization of Infinite Dimensional Systems with Applications, Springer Science \& Business Media (1999).

36. Huebner, K.H., Dewhirst, D.L., Smith, D.E., and Byrom, T.G., The Finite Element Method for Engineers, John Wiley \& Sons (2008).

37. Gad-el-Hak, M., MEMS: Introduction and Fundamentals, CRC press (2005).

\section{Biographies}

Amin Mehrvarz received his BSc and MSc degrees both in Mechanical Engineering from Sharif University of Technology (SUT), Tehran, Iran in 2013 and 2015, respectively. His current research interests include nonlinear systems and control, PDE control, special purpose robotics and intelligent machines, vibration 
analysis and control of distributed parameter systems and vehicle control.

Hassan Salarieh received his BSc degree in Mechanical Engineering and also Pure Mathematics from Sharif University of Technology, Tehran, Iran in 2002. He graduated from the same university with MSc and $\mathrm{PhD}$ degrees in Mechanical Engineering in 2004 and 2008. At present, he is an Associate Professor in Mechanical Engineering at Sharif University of Technology. His fields of research are dynamical systems, control theory, and stochastic systems.

Aria Alasty received his BS and MS degrees in Mechanical Engineering from Sharif University of Technology (SUT), Tehran, Iran in 1987 and 1989, respectively, and his PhD degree in Mechanical Engineering from Carleton University, Ottawa, Canada in 1996. At present, he is a Professor of Mechanical Engineering at SUT. He has been a member of the Center of Excellence in Design, Robotics, and Automation (CEDRA) since 2001. His main areas of research include nonlinear and chaotic systems control, computational nano/micro mechanics and control, special-purpose robotics, robotic swarm control, and fuzzy system control.

Ramin Vatankhah received his BS degree in Mechanical Engineering from the Shiraz University, Shiraz, Iran in 2007. He received his MS and PhD degrees in Mechanical Engineering from the Sharif University of Technology, Tehran, Iran in 2009 and 2013. He is currently an Assistant Professor of the Department of Mechanical Engineering at Shiraz University. His research interests include nonlinear vibration, chaos control, evolutionary computation techniques, boundary control of partial differential equations, nonlinear control, and fuzzy system control. 